CARDIORESPIRATORY FITNESS BENEFITS VERBAL MEMORY IN OLDER BUT NOT YOUNGER BREAST CANCER PATIENTS

H. Derry, W.B. Malarkey, J. Kiecolt-Glaser, Institute for

Behavioral Medicine Research, The Obio State University

Wexner Medical Center, Columbus, Ohio

Breast cancer survivors often experience cognitive problems following surgery and prior to further treatment. Cardiorespiratory fitness (CRF) benefits cognitive function in healthy older adults. However, few studies address CRF's effects among breast cancer survivors or consider how this pattern may vary by age. As part of a larger study, 110 female post-surgical breast cancer survivors (ages 26 to 75, stages I-IIIA) completed a graded cycle ergometry test to measure peak oxygen consumption prior to chemotherapy or radiation treatment. Neuropsychological tasks assessed verbal memory (Hopkins Verbal Learning Test), verbal fluency (F-A-S), executive function (Trail Making Test, n-back task), and sustained attention (Continuous Performance Test). Regression models revealed that the relationship between CRF and immediate verbal recall scores varied significantly by age, above and beyond education, comorbidities, cancer stage, and time since surgery $(b=.013, \mathrm{SE}=.006, \mathrm{p}=.032)$. Among women ages 62 and older, those with better fitness had better immediate verbal recall compared to those with poorer fitness, using the Johnson-Neyman method. The age by CRF interaction did not significantly predict performance on other cognitive tasks in adjusted models. Accordingly, older participants who were more fit had better immediate verbal recall than those who were less fit, while younger women's CRF was not significantly related to their verbal memory performance. The fitness-cognitive function link may be particularly relevant for older cancer survivors compared to their younger counterparts. Longitudinal studies would help discern if physical fitness prevents accelerated cognitive aging among cancer survivors, and prompt relevant interventions.

\section{FACTORS SHAPING GRANDPARENTS RESPONSIBILITY FOR GRANDCHILDREN IN THREE- GENERATION HOUSEHOLDS}

N. Velasco, J.E. Mutchler, Gerontology, University of

Massachusetts Boston, Boston, Massachusetts

The number of grandparents identified as primary responsible for grandchildren is on the upswing. Data from the American Community Survey (ACS) suggest that the percent of coresident grandparents responsible for grandchildren increased from $11 \%$ in 2010 to $15 \%$ in 2015 . Additionally, estimations indicate that among the nearly 2.7 million U.S. grandparents who claimed responsibility for their minor grandchildren in 2015, two-thirds lived in a three-generation household, including minor grandchildren, one or both of their parents, and one or more grandparents. However, most of the literature on grandparent caregivers has focused on the grandparent's role in skipped-generation households, and little is known about the grandparent's role in three-generation households. The purpose of this study is to explore the factors that lead grandparents to be identified as primarily responsible for grandchildren living with them in three-generation households. The analysis was conducted using the 2015 ACS 5-year estimates dataset. Household income and income balance between the grandparent and parent generations are determinants of whether the grandparent is classified as being primarily responsible; specifically, grandparents who provide a high share of household income are more likely to be classified as primarily responsible for the grandchild. As well, race and ethnicity are related to the grandparent's identification as primary caregiver, with African American grandparents being more likely to be classified as primarily responsible for grandchildren. Together, these findings suggest that a grandparent's responsibilities in the three-generation household are defined in part by economic responsibility, but in part by cultural norms relating to the grandparent role.

\section{MANIPULATION OF EFFICACY AND FUNCTIONAL MOTOR PERFORMANCE IN OLDER WOMEN}

N. Roncesvalles ${ }^{1}, \mathrm{M}$. Bregendahl ${ }^{2}, 1$. Texas Tech University, KInesiology and Sport Management, Lubbock, Texas,

2. Stanford University School of Medicine, Division of Primary Care, Palo Alto, California

Fall prevention remains a worthy goal for the aging population (Ungar, et al., 2013). This study contributes by testing the malleability of motivation and behavior of older women beginning to exhibit the fear of falling. Will manipulation of efficacy significantly affect both perceived fear (falls efficacy) and behavior (i.e. gait, balance)?

Forty-two older women ( $\mathrm{N}=42)$, between 55 to 75 years were administered with the Falls Efficacy Scale-International (FES-I) and the Short Physical Performance Battery (SPPB) on two occasions: 1) Initial screening (baseline), and, 2) Postmanipulation. Baseline assessment included documentation of health, mental state, heart rate, activity levels and other demographic data. Those with low to moderate concern for falling (FES-I) were included in the participant pool and randomly assigned to three groups: enhanced efficacy (EE), reduced efficacy (RE), and control (C). A rhythmic weight shifting task (SMART Balance Master ${ }^{\circledR}$ ) was undertaken to manipulate perception (efficacy). Fabricated verbal feedback were tailored according to group: regardless of actual performance, positive for EE, and negative for RE. Participants in the C group were provided none. Comparison (Friedman's Test) of the pre- and post-manipulation scores revealed that provision of manufactured verbal feedback did not influence FES-I or SPPB in any group examined $(\mathrm{p} \geq 0.05)$. In addition, no significant relationship was found between the variables before and after manipulation.

The non-significant results were likely attributable to the short intervention protocol. Perhaps a longer and consistent protocol, with specific focus on populations already experiencing a significant level of fear will produce alternative results.

\section{DEPRESSIVE SYMPTOMS IN CHILD CAREGIVERS OF VERY OLD MEXICAN AMERICANS}

D.V. Flores ${ }^{1}$, S. Rote ${ }^{2}$, J.L. Angel ${ }^{3}$, N. Chen ${ }^{1}$, B. Downer ${ }^{1}$, K.S. Markides ${ }^{1}, 1$. University of Texas Medical Branch, Galveston, Texas, 2. University of Louisville, Kent School of Social Work, Louisville, Kentucky, 3. University of Texas, Austin, Texas

Objective: To study the effects of disability, cognitive impairment, and neuropsychiatric disturbance among older 
Mexican Americans on depressive symptoms in their children caregivers.

Methods: This study utilizes data from Wave 7 (2010-11) of the Hispanic Established Populations for the Epidemiologic Study of the Elderly to assess caregivers that provided direct personal care with activities of daily living (ADL) and who were children of the care recipient. Two hundred adult caregivers provided direct personal care (e.g., bathing, toileting, dressing, etc.) to their older parents (average age $=87$ ). We analyzed the influence of ADL disability, cognition (MMSE), and neuropsychiatric symptoms (NPI) on depressive symptoms of the adult child caregiver. A cross-sectional multivariable linear regression analysis was conducted to examine the effect of neuropsychiatric disturbance on caregiver depressive symptoms.

Results: ADL disability of the care recipient, cognitive functioning of the care recipient, and caregiver health status alone did not have a significant effect on depressive symptoms of the caregiver but NPI of the care-recipient did. Not being married, high perceived social stress, and caregiverassessed NPI of the care recipient had a significant effect on caregiver depressive symptoms.

Conclusions: In a Mexican American familistic culture, disability and cognitive impairment might be better tolerated by families but neuropsychiatric behavioral symptoms related to dementia may take an increased toll on family member caregivers. The need to provide respite services, mental health resources and community services for caregivers of care recipients with neuropsychiatric dysfunction is of paramount importance to alleviate depressive symptoms and burden among caregivers.

\section{THE STRENGTH OF SOCIAL TIES: HOW COUPLES FACE AD TOGETHER OR ALONE}

R.L. Beard ${ }^{1}$, R. Daley' ${ }^{2}$, M. O'Connor ${ }^{2}$, S. Shirk ${ }^{2}, 1$. Sociology and Anthropology, College of the Holy Cross, Worcester, Massachusetts, 2. Edith Nourse Rogers Memorial Bedford VAMC, Beford, Massachusetts

Social and behavioral research on Alzheimer's disease (AD) has largely neglected the potential positive appraisals of $\mathrm{AD}$ experiences relative to the so-called stress and burden assumed to accompany the condition. The very word caregiving implies a unidirectional and exclusively negative experience for everyone involved. Spouses provide the majority of care for individuals with $\mathrm{AD}$ yet few prior studies have examined the association between positive caregiving appraisals and quality of marital relationship. The qualitative literature suggests that these couples adopt either a We/ Us approach where they describe experiences as a composite whole or an I/Me approach where they describe themselves as experiencing the impact of $\mathrm{AD}$ separately. Little is known about how these perspectives relate to the individual characteristics of either party. Eleven spousal dyads were divided into $\mathrm{I} / \mathrm{Me}(\mathrm{n}=5)$ and We/Us $(\mathrm{n}=6)$ groupings based on qualitative analyses. Diagnosed individuals were given measures of cognitive and functional ability and caregivers completed anxiety, depression, burden, relationship satisfaction, and positive aspects of caregiving measures. We found no significant differences between groups on patient cognitive or functional ability, or caregiver anxiety, depression, burden, or relationship satisfaction. However, We/Us caregivers expressed more positive aspects of caring than I/Me couples. These findings suggest the I/Me approach is not associated with differences in cognitive status or functional ability or carer emotional health, perceived burden, or relationship satisfaction. Those taking a We/Us approach, however, were able to identify more positive aspects of caring. This may be related to mutual compassion and could be protective.

\section{PASSIVE SUICIDAL IDEATION AMONG OLDER ADULT INSOMNIA CLINIC PATIENTS WITH INSOMNIA DISORDER}

R. Cui, D. Richter, A. Fiske, Psychology, West Virginia

University, Morgantown, West Virginia

Up to $29 \%$ of older adults suffer from insomnia symptoms (Ancoli Israel \& Cooke, 2005; Schubert et al., 2002). Symptoms of insomnia have been found to be associated with suicidal ideation among older adults (Nadorff, Fiske, Sperry, Petts \& Gregg, 2013). The present study seeks to investigate factors associated with suicidal ideation within a sample of 51 older adults diagnosed with Insomnia Disorder seeking treatment at an Insomnia Clinic. The sample was primarily female $(70.6 \%)$ and Caucasian $(96.1 \%)$. Participants completed self-report measures of insomnia symptoms (Insomnia Severity Index, ISI), daytime sleepiness (Epworth Sleepiness Scale, ESS), anxiety symptoms (Beck Anxiety Inventory, BAI), depression symptoms (Center for Epidemiologic Studies Depression Scale Revised, CESD-R), emotion regulation strategies (Emotion Regulation Questionnaire reappraisal and suppression scales, ERQ reappraisal and ERQ suppression), and social support (Interpersonal Support Evaluation List, ISEL). Passive suicidal ideation was assessed with items 14, "I wished I were dead" and 15, "I wanted to hurt myself", from the CESD-R and coded as a dichotomous variable. Passive suicidal ideation was endorsed by $9.8 \%$ of the sample. Older adults with passive suicidal ideation had more anxiety symptoms $(\mathrm{F} 1,48=17.59, \mathrm{p}<.01)$, more depression symptoms $(\mathrm{F} 1,49=21.39, \mathrm{p}<.01)$, greater use of suppression strategies to regulate negative emotions $(\mathrm{F} 1,36=7.24$, $\mathrm{p}=.011)$, and less social support $(\mathrm{F} 1,31=6.37 \mathrm{p}=.017)$. These findings indicate the need to screen for suicidal ideation among older adults seeking treatment for insomnia who present with elevated depression or anxiety symptoms.

\section{MINDFULNESS-BASED PROGRAM ENHANCES THE QUALITY OF LIFE FOR COMMUNITY-DWELLING OLDER ADULTS IN TAIWAN}

H. Hsu ${ }^{1}$, S. Cheng ${ }^{1}$, Y. Hsu ${ }^{1}$, C. Chiu ${ }^{1}$, C. Kuo 2 , 1. Yangsheng Foundation, Taipei, Taiwan, 2. National Pingtung University, Pingtung, Taiwan

Aim: This study aimed to evaluate the impact of an eight weeks mindfulness-based program on the quality of life for older adults form community population.

Methods: The benefits of mindfulness research on human well-being have accumulated much empirical evidences. Several studies have also indicated that mindfulness training enhances cognitive functions. However, it is unknown whether the positive effect can be generalized to older adults. An eight-weeks mindfulness-based program which was modified from Mindfulness-based Cognitive Therapy(MBCT) that involve understanding mindfulness, the benefits of mindfulness to increase self-awareness, 